\title{
Temperature and Voltage Offsets in High-ZT Thermoelectrics
}

\author{
GEORGE S. LEVY ${ }^{1,2}$ \\ 1.—Entropic Power, 130 Rockview, Irvine, CA 92612, USA. 2.—e-mail: glevy@entropicpower.com
}

Thermodynamic temperature can take on different meanings. Kinetic temperature is an expectation value and a function of the kinetic energy distribution. Statistical temperature is a parameter of the distribution. Kinetic temperature and statistical temperature, identical in Maxwell-Boltzmann statistics, can differ in other statistics such as those of Fermi-Dirac or BoseEinstein when a field is present. Thermal equilibrium corresponds to zero statistical temperature gradient, not zero kinetic temperature gradient. Since heat carriers in thermoelectrics are fermions, the difference between these two temperatures may explain voltage and temperature offsets observed during meticulous Seebeck measurements in which the temperature-voltage curve does not go through the origin. In conventional semiconductors, temperature offsets produced by fermionic electrical carriers are not observable because they are shorted by heat phonons in the lattice. In high-ZT materials, however, these offsets have been detected but attributed to faulty laboratory procedures. Additional supporting evidence for spontaneous voltages and temperature gradients includes data collected in epistatic experiments and in the plasma Q-machine. Device fabrication guidelines for testing the hypothesis are suggested including using unipolar junctions stacked in a superlattice, alternating $n / n^{+}$and $p / p^{+}$junctions, selecting appropriate dimensions, doping, and loading.

Key words: Statistical mechanics, non-Maxwellian, thermoelectric, Seebeck offset, second law, nontransitive

\section{INTRODUCTION}

This paper discusses mysterious voltage and temperature offsets observed during very precise and careful measurement of the Seebeck effect in thermoelectric materials, ${ }^{1}$ as shown in Fig. 1a. Sometimes, the Seebeck curve does not go through the origin, as shown in Fig. $1 \mathrm{~b}$, indicating a temperature difference output without any voltage input, or a voltage output without any temperature difference input (see Supplementary Material for experimental details).

(Received July 4, 2017; accepted October 10, 2017; published online October 25, 2017)
Such data appear to violate the second law and are therefore conventionally assumed to be caused by poor laboratory practices. Many sources of error have been identified and mitigated, ${ }^{1-3}$ as presented in Table I.

Yet these unexplained and irreducible offsets persist despite the most meticulous efforts by researchers $^{1-4}$ to eliminate them. This effect is viewed as a nuisance and commonly forcefully removed from the data by taking difference measurements (i.e., the slope method). ${ }^{2-4}$

Do poor laboratory practices cause offsets? Definitely. Is there any other reason, independent of experimental errors, that could also produce offsets? This is the question addressed herein. The goal is to present a hypothesis that explains experimentally irreducible offsets, and to show how this hypothesis conforms with the second law as currently understood. 


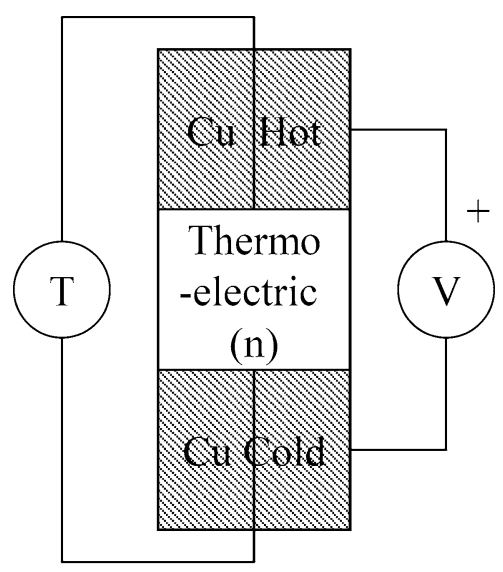

(a)

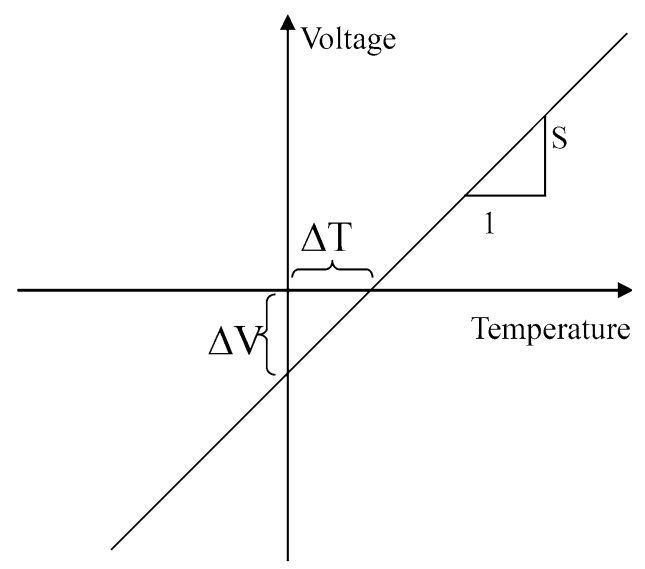

(b)

Fig. 1. (a) Seebeck measurement of thermoelectric material. (b) Seebeck curve not passing through the origin.

Table I. Sources of error and mitigation measures in Seebeck measurements

\section{Source of error}

Poor thermal connectivity

Lack of colocation and simultaneity in $T$ and $V$ measurements Spurious Seebeck voltages between the $V$ probe and the sample Chemical reactivity between thermocouple and sample

Lack of steady state, thermal drift

\section{Mitigation measure}

Spring-loaded thermal contacts

Better geometry (uniaxial configuration)

$V$ probe with low Seebeck coefficient (e.g., tungsten) Shield thermocouple with inert material such as graphite Algorithm, filters

\section{WHAT IS TEMPERATURE?}

The foregoing analysis requires a clear understanding of temperature in the context of thermoelectric materials. Consider the general case of a gas subjected to a force field, as shown in Fig. 2.

Let us assume that the kinetic energy $E_{\mathrm{k}}$ of the gas particles follows a particular statistical distribution $f\left(E_{\mathrm{k}}, E_{\mathrm{p}}, \theta_{T}\right)$, where $E_{\mathrm{p}}$ is the potential energy and $\theta_{T}$ is the temperature. Since the gas column forms a single statistical ensemble, $\theta_{T}$ is invariant with elevation (as per the second law). The expected value for the kinetic energy of a gas particle is

$$
<E_{k}>=\int_{0}^{\infty} E_{\mathrm{k}} f\left(E_{\mathrm{k}}, E_{\mathrm{p}}, \theta_{T}\right)_{\text {normalized }} \mathrm{d} E_{\mathrm{k}} .
$$

Temperature can also be expressed as a function of the expected kinetic energy:

$$
T\left(E_{\mathrm{p}}, \theta_{T}\right)=\frac{2}{3 k_{\mathrm{B}}} \int_{0}^{\infty} E_{\mathrm{k}} f\left(E_{\mathrm{k}}, E_{\mathrm{p}}, \theta_{T}\right)_{\text {normalized }} \mathrm{d} E_{k} .
$$

In conventional thermodynamics, $\theta_{T}$ and $T$ are both called "thermodynamic temperature." This nomenclature is adequate when these two temperatures are identical, as it happens to be when the distribution is Maxwell-Boltzmann. However, as explained below, $\theta_{T}$ and $T$ can be different when the distribution is not Maxwellian (e.g., biased halfMaxwellian, ${ }^{5}$ Fermi-Dirac, ${ }^{6-8}$ or Bose-Einstein).

\section{Fermi-Dirac gas}

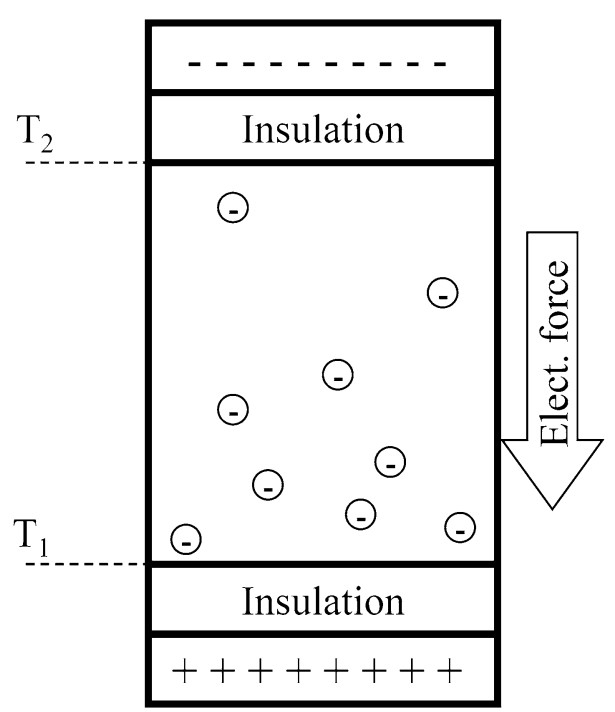

Fig. 2. Gas subjected to a force. A low-density enclosed gas is subjected to an external force. If the gas particles are charged carriers, as found in a thermoelectric material, then the force can be an electrical field.

The term "thermodynamic temperature" becomes confusing as it refers to two different quantities. Therefore, for the purpose of this paper, this term shall be avoided. The more precise term "statistical 
temperature" shall be reserved to express $\theta_{T}$ because it is a parameter of a statistical distribution. "Kinetic temperature" shall be reserved for $T$ because it is proportional to the expected kinetic energy of the particles. The distinction between these two temperatures is not entirely new. ${ }^{9,10}$

If one considers the gas as a fully connected thermodynamic ensemble with a single distribution, then obviously $\theta_{T}$ is always invariant with elevation, independent of the distribution itself. In other words, $\theta_{T}$ is always constant whether the gas is Maxwellian or comprises fermions or bosons. This is consistent with the second law and confirmed by a proof by Tolman in Ref. 11 (pages 551, 552). The question is whether $T$ is also constant with elevation. Two cases shall be considered:

1. A Maxwellian gas

2. A fermion gas.

\section{Maxwellian Gas}

The distribution for a Maxwellian gas is ${ }^{6,7}$ :

$$
\begin{aligned}
& f_{\mathrm{MB}}\left(E_{\mathrm{k}}, E_{\mathrm{p}}, \theta_{T}\right) \\
& \quad=2\left(\frac{1}{k_{\mathrm{B}} \theta_{T}}\right)^{3 / 2} \sqrt{\frac{E_{\mathrm{k}}}{\pi}} \exp \left(\frac{-E_{\mathrm{k}}}{k_{\mathrm{B}} \theta_{T}}\right) \exp \left(\frac{-E_{\mathrm{p}}}{k_{\mathrm{B}} \theta_{T}}\right) .
\end{aligned}
$$

The term $E_{\mathrm{p}}$ expresses the potential energy of the particles. This term reduces the amplitude of the distribution, indicating a decrease in the density of the particles with elevation. However, it also denormalizes the distribution. Renormalizing Eq. 3 for a given elevation eliminates $E_{\mathrm{p}}$ (because it is constant), ${ }^{6,7}$ resulting in

$$
f_{\mathrm{MB}}\left(E_{\mathrm{k}}, \theta_{T}\right)_{\text {normalized }}=2\left(\frac{1}{k_{\mathrm{B}} \theta_{T}}\right)^{3 / 2} \sqrt{\frac{E_{\mathrm{k}}}{\pi}} \exp \left(\frac{-E_{\mathrm{k}}}{k_{\mathrm{B}} \theta_{T}}\right) .
$$

In other words, the distribution is the same at ground level or at any given elevation, indicating that the normalized Maxwell-Boltzmann distribution is independent of elevation.

\section{Fermion Gas}

If the gas particles are fermions, their distribution is Fermi-Dirac (FD) and given by ${ }^{6,7}$

$$
\begin{aligned}
& f_{\text {Fermions }}\left(E_{\mathrm{k}}, E_{\mathrm{p}}, \theta_{T}\right)_{\text {normalized }} \\
& \quad=A\left(E_{\mathrm{p}}, \theta_{T}\right) \sqrt{E_{\mathrm{k}}} \frac{1}{1+\exp \left(\frac{E_{\mathrm{k}}+E_{\mathrm{c}}+E_{\mathrm{p}}-E_{\mathrm{F}}}{k_{\mathrm{B}} \theta_{T}}\right)} .
\end{aligned}
$$

Introducing the potential energy term $E_{\mathrm{p}}$ denormalizes the distribution, but renormalization does not eliminate $E_{\mathrm{p}}$ because the expression is not strictly exponential. The difference between the Maxwellian gas and a fermion gas is summarized in Fig. 3.
Maxwellian gases are statistically invariant with elevation, and therefore comply with the second law.

$$
T=\theta_{T}=\text { constant with elevation. }
$$

In non-Maxwellian gases, however, the first moment of the distribution changes with elevation. More particularly, in fermion gases, this moment decreases, indicating that

$$
\frac{\mathrm{d} T}{\mathrm{~d} z}<0
$$

The hypothesis proposed by the author is that semiconductor junctions develop a temperature gradient in addition to their built-in potential. In conventional semiconductors, this temperature gradient is quickly shorted by heat phonons but has been inadvertently observed as voltage and temperature offsets in high-performance (ZT) thermoelectric materials in which the coupling between electrical carriers and phonons is low. These offsets have been observed, published, ${ }^{13}$ and dismissed as due to poor laboratory practices. Yet they do not go away.

Does a spontaneous temperature gradient in a non-Maxwellian gas, for example, electrical carriers in a thermoelectric material, have an explanation within the domain of applicability of the second law? This topic is discussed in the next section.

\section{NON-MAXWELLIAN SYSTEMS}

Two kinds of systems shall be discussed:

1. Homogeneous non-Maxwellian systems

2. Heterogeneous non-Maxwellian systems.

\section{Homogeneous Non-Maxwellian Systems}

Spontaneous voltages are well known; For example, a semiconductor junction has a built-in potential. This potential complies with the second law as currently understood because it is inaccessible for energy production. If one were to connect leads to either side of the junction, the contacts between the leads and semiconductor would produce a voltage exactly canceling the built-in potential. Two reasons can be invoked for this exact cancellation:

1. The electrons in the leads are subjected to the same field as the electrons in the semiconductor. The electrical field is scalar, therefore a loop summation of voltage around the lead and semiconductor is exactly zero. No energy can be extracted.

2. The electrons are all identical to each other and comply with the assumption of homogeneity and indistinguishability of the $H$-theorem, ${ }^{14-18}$ which states that entropy can never decrease. Therefore, the second law is upheld. No energy can be extracted. 


\begin{tabular}{|c|c|c|}
\hline & MB+Field $\quad \begin{array}{l}\text { denormalizes } \\
\text { distribution }\end{array}$ & FD+Field (Thermoelectrics) $\begin{array}{l}\text { denormalizes } \\
\text { distribution }\end{array}$ \\
\hline Distribution & $f_{M B}=2\left(\frac{1}{k_{B} \theta_{T}}\right)^{3 / 2} \sqrt{\frac{E_{k}}{\pi}} \frac{1}{\exp \left(\frac{E_{k}+E_{p}}{k_{B} \theta_{T}}\right)}$ & $f_{\text {Fermions }}=A \sqrt{E_{k}} \frac{1}{1+\exp \left(\frac{E_{k}+E_{c}-E_{F}+E_{p}}{k_{B} \theta_{T}}\right)}$ \\
\hline$E_{P}=0$ & normalized distribution & lized distribution \\
\hline$E_{P}>0$ & $\begin{array}{r}\text { renormalized distribution } f_{M B \text { Normalized }}\left(E_{P}>0\right) \\
=f_{M B \text { Normalized }}\left(E_{P}=0\right) \\
E_{P} \text { eliminated by } \\
\mathrm{E}_{\mathrm{K}} \quad \text { renormalization }\end{array}$ & 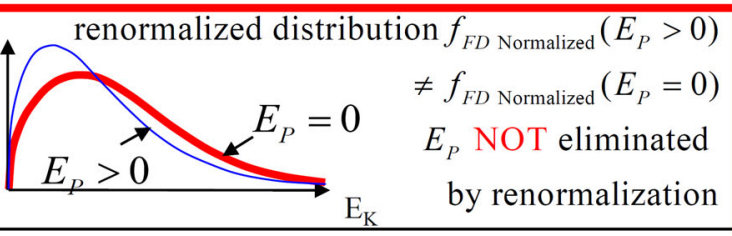 \\
\hline & $T=\theta_{T} ; \frac{d T}{d z}=0 ;$ column is isothermal & $T \neq \theta_{T} ; \frac{d \theta_{T}}{d z}=0 ; \frac{d T}{d z}<0$ \\
\hline
\end{tabular}

Fig. 3. Maxwell-Boltzmann (MB) gases remain isothermal with elevation. Non-MB such as Fermi-Dirac (FD) gases change kinetic temperature with elevation. These graphs have been generated by a calculator program. ${ }^{12}$

As explained above, a kinetic temperature gradient occurs spontaneously in fermion gases subjected to a field. However, statistical temperature remains invariant with elevation because the gas constitutes a single thermodynamic ensemble with a single distribution parametrized by $\theta_{T}$. Therefore, the second law is upheld if expressed in terms of $\theta_{T}$ not $T$. This is confirmed by the proof by Tolman in Ref. 9 (pages 551, 552), who shows that heat flows down the statistical temperature gradient.

Can the kinetic temperature gradient across a thermoelectric junction be used to produce energy, for example, by connecting a heat engine (a Seebeck device) across a junction? The same argument used for the built-in potential in the semiconductor example can be applied, as shown in Fig. 4.

If the heat carriers ferrying heat to the heat engine are homogeneous with and indistinguishable from the heat carriers in the thermoelectric junction, then the carriers develop the same kinetic temperature gradient as the junction and no energy can be produced. This is confirmed by the $H$-theorem, which assumes homogeneity and indistinguishability of particles.

\section{Heterogeneous Non-Maxwellian Systems}

Heterogeneous systems fall outside the $H$-theorem because they comprise particles with different statistics. The author ${ }^{8}$ explains how the second law can be generalized to cover such systems. Statistical temperature is more accurate than kinetic temperature in expressing thermodynamic processes. Clausius' formulation of the second law ${ }^{19,20}$ requires heat to flow down the statistical temperature gradient, not necessarily down the kinetic temperature gradient. Boltzmann's formulation of the second law (the $H$-theorem-entropy never decreases) remains correct if entropy is expressed using statistical temperature. Other formulations of the second law by Planck and Kelvin completely break down. Heat can be converted to work.

Figure 5 provides an example of a heterogeneous system. A fermion column (e.g., electrons in a thermoelectric) is subjected to a vertical electrical field and thermally connected to a phonon column (e.g., heat carriers in a metal). The fermions are susceptible to the electric field and therefore produce a spontaneous temperature gradient because of their statistics. The heat phonons are not affected by the field and therefore do not produce any temperature difference. Unlike the homogeneous case, the fermion temperature gradient is not actively cancelled, it is passively shorted by the phonons, resulting in a circular heat flow, up the phonon column and down the fermion column. A heat engine (i.e., a Seebeck device) inserted into the thermal path can convert heat to work. In fact, simply measuring a temperature difference using a thermocouple implies that heat is converted to work.

Voltage and temperature offsets are due to the difference between statistical temperature and kinetic temperature in heterogeneous systems, and result in conversion of heat to work. This challenge to the second law is not an isolated phenomenon in physics and has led the author to formulate a generalization of the second law. ${ }^{8}$ Other such phenomena include: 


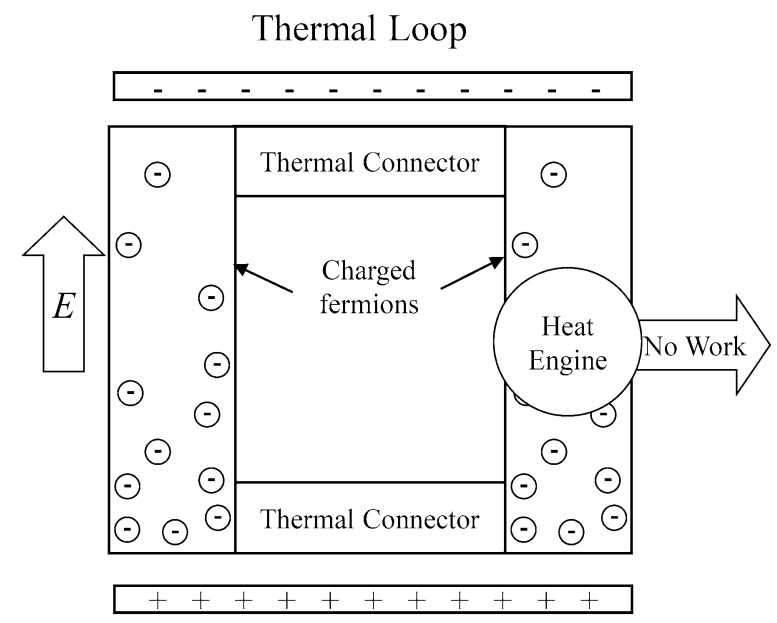

(a)
Electrical Loop

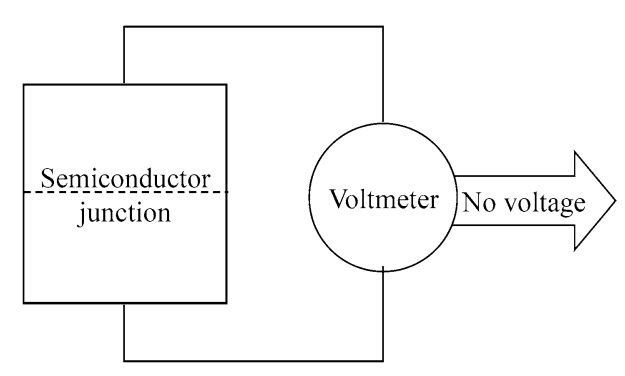

Fig. 4. (a) In a homogeneous system, the temperature gradient produced by a thermoelectric material subjected to a field is exactly cancelled by the temperature gradient generated by the thermal connection to the engine. The temperature difference cannot be used to produce work. (b) The phenomenon is analogous to the impossibility of producing energy from the built-in potential in a semiconductor junction.

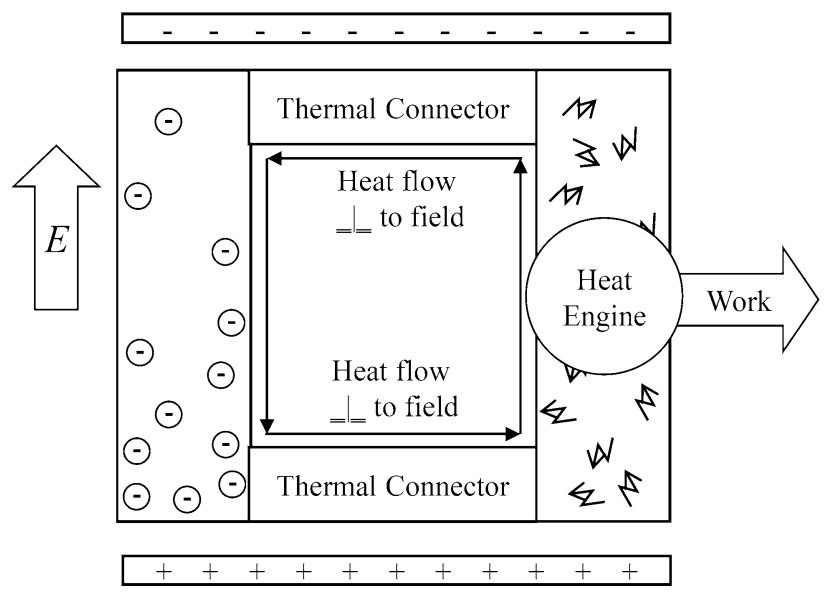

Fig. 5. The temperature gradient generated by the fermion column is not cancelled by heat phonons. A heat engine can exploit this temperature gradient to convert heat to work.

The reciprocal Hall effect ${ }^{5}$ : In the presence of a magnetic field parallel to a surface, and an electric field perpendicular to the surface, electrical carriers at the surface generate a voltage perpendicular to both fields and parallel to the surface. This effect relies on the biased half-Maxwellian statistics generated at the surface by electrical carriers that follow interrupted cyclotron orbits.

Epicatalysis ${ }^{21-25}$ : This phenomenon has been experimentally verified in the laboratory by Sheehan. It refers to a catalytic reaction that shifts the equilibrium of the reaction, thereby violating detailed balance.

$\mathrm{Q}$ machine $\mathrm{e}^{26,27}$ : This is a plasma device that also exhibits well-documented epicatalytic behavior.

Unpowered light-emitting diode (LED) emission. Light emission from unbiased LEDs has been experimentally demonstrated by Orem. ${ }^{28}$

\section{EXPERIMENTAL TEST OF HYPOTHESIS}

Offsets in thermoelectric measurements may be caused by a genuine physical phenomenon, and therefore could represent an important source of energy. This hypothesis can be tested in the laboratory using the architecture illustrated in Fig. 6 and the following guidelines.

Traditionally, voltage and temperature offsets are undesirable, and forcefully eliminated (using, e.g., the slope method $^{2,4}$ ). In contrast, the following guidelines aim at maximizing these offsets:

1. Heat carriers should be non-Maxwellian, which is of course fulfilled by the electrons or holes (i.e., fermions) in a semiconductor material.

2. A force field is required. This can be produced by the built-in potential across the semiconductor junction or by placing the semiconductor between insulated capacitor plates.

3. Unlike the Peltier effect, the proposed effect is induced by a field, not by a current. Therefore, if a junction is used, it should be insulated to prevent any current passing through it, as shown in Fig. 6.

4. The material should have high $Z T$ to minimize coupling between carriers and heat phonons, thereby reducing thermal short circuits by the phonons. In other words, the carriers should behave mostly ballistically with respect to the lattice.

5. The carriers should be of a single polarity, either electrons or holes, but not both as in a bipolar junction. Carriers of opposite charge would produce thermal gradients in opposite directions, canceling each other. Therefore, if a junction is used, it should be unipolar. 
6. Stacking of temperature difference generators such as in a superlattice can amplify the overall output temperature difference.

7. Stacking and judicious alternation in the layer materials can also reinforce the electrical field across each layer of the stack, leading to a stronger effect, for example, insulator $/ n / n^{+} /$insulator $/ p / p^{+} /$insulator $/ n / n^{+} /$ insulator $/ p / p^{+} / \ldots /$ insulator. This pattern allows fields from adjacent layers to reinforce.

8. The number of carriers per unit surface of the junction should be low enough to avoid generating a space charge that would cancel the electric field. The number of carriers per unit surface can be controlled by adjusting the thickness of the thermoelectric layer. In other words, the device should be thin enough and have moderate doping to operate approximately in the depletion zone.

9. The carrier density should be high enough for the carriers to develop their own nonMaxwellian distribution independently of the lattice. This can be controlled by lowering the temperature near or below the Fermi temperature, and increasing doping. This requirement and the previous one imply a trade-off in doping.

10. The material diffusion zone produced by the junction manufacturing process should be significantly smaller than the electronic depletion zone defined by the carriers during operation of the device.

11. Connecting a Seebeck device across a temperature difference generator produces electrical power in proportion to the temperature difference $\Delta T$ applied across the device. A number $n$ of such electrically connected combinations produces power in proportion to $n \Delta T$. However, connecting a single Seebeck device across a stack of $n$ temperature difference generators increases the temperature difference input across the Seebeck device from $\Delta T$ to $n \Delta T$ and furthermore increases the Carnot efficiency of the device by $n$, resulting in a power output proportional to $n^{2} \Delta T$. Therefore, in the absence of any other design constraints (e.g., geometry, minimum and maximum operating temperatures, heat sinks, etc.), the preferred architecture is a single Seebeck device connected to a stack of temperature difference generators.

The last guideline that recommends maximizing Carnot efficiency is illustrated in Fig. 7. The device in Fig. 7a shows several Seebeck devices, each connected across a single temperature difference generation device. The power output of such an arrangement as shown in Fig. $7 \mathrm{~b}$ is proportional to the number $n$ of such modules. However, if a single

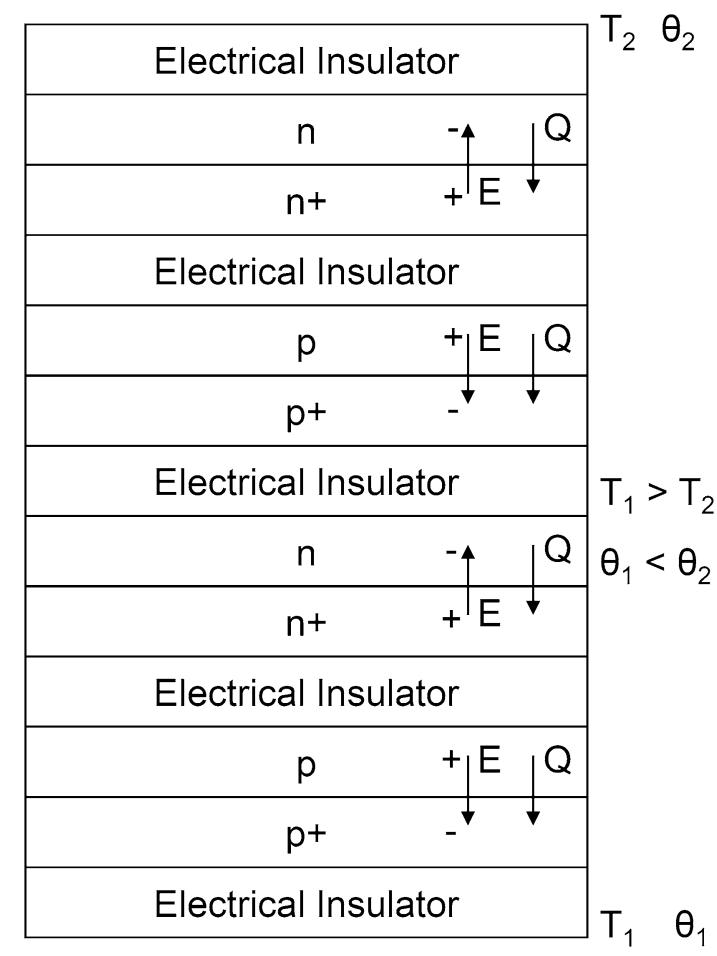

Fig. 6. Proposed architecture for testing proposed hypothesis. Superlattice comprising interleaved and insulated homogeneous $\mathrm{n} /$ $n^{+}$junctions and $p / p^{+}$junctions. The junctions are arranged such that their built-in fields reinforce each other. $E$ represent the electric field and $Q$ represents the heat flow.

Seebeck device is connected across a stack of $n$ multiple temperature difference generators, as illustrated in Fig. 7c, the power output becomes proportional to $n^{2}$, as shown in Fig. 7d.

Why not operate the device directly as an electrical generator? In other words, use the voltage offset rather than the temperature offset. To do this, the temperature offset needs to be thermally short circuited, but the resulting efficiency is then limited by the Carnot limit as determined by the temperature offset of an individual junction, which is typically small. [The experiment by Iwanaga et al. ${ }^{1}$ (see Supplementary Material) indicates a temperature offset of about $2^{\circ} \mathrm{C}$.] As shown in Fig. 7, this limitation can be improved by boosting the available temperature difference seen by the Seebeck device. It is better to connect a single Seebeck device across a stack of $n$ junctions than to connect $n$ Seebeck devices in series, each across a single junction. In the former case, the power output is proportional to $n^{2}$, whereas in the latter it is proportional to $n$ because of the Carnot limit.

Variations on the basic design of Fig. 6 are possible; For example, one can use the built-in potential of heterojunctions instead of homojunctions, as shown in Fig. 8a. Alternatively, one could use a stack of capacitive thin plates as shown in Fig. 8b. A significant disadvantage of the capacitor designs in Fig. 8b compared with the junction 


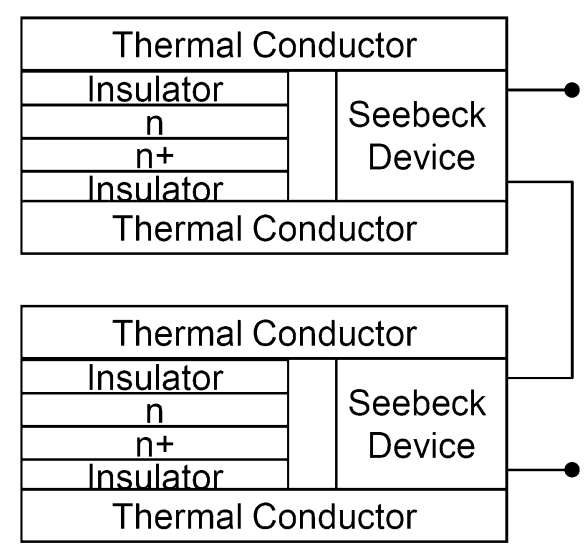

(a)

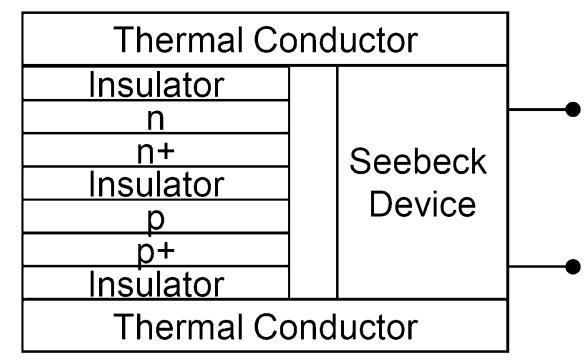

(c)

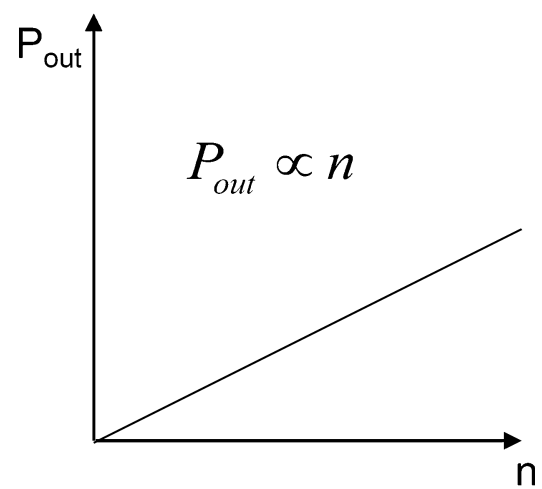

(b)

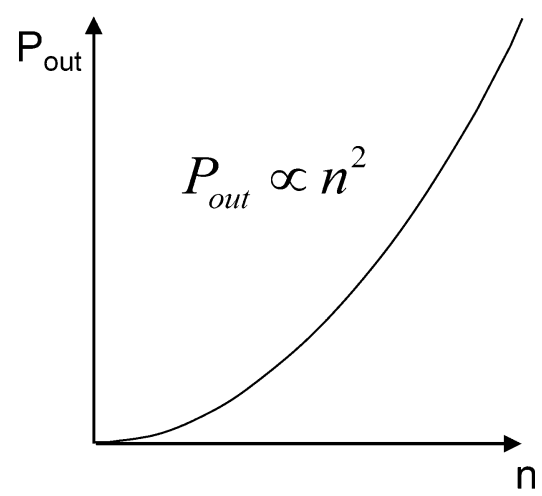

(d)

Fig. 7. (a) Multiple Seebeck devices connected in series, each device across a single layer temperature generator. (b) Power output is proportional to the number $n$ of Seebeck devices. (c) Single Seebeck device across a stack of temperature generators. (d) Power output is proportional to $n^{2}$, where $n$ is the number of stack elements.

designs shown in Figs. 6 and $8 \mathrm{a}$ is the risk of catastrophic breakdown of the capacitors.

Some of the guidelines require tradeoffs between design parameters. These parameters include:

1. Doping level

2. Ionization energy of selected dopant

3. Thickness of thermoelectric layers

4. Thickness of the junction material diffusion zone.

The following series of steps provides a method for performing the required trade-offs:

1. Select a high-ZT material.

2. Determine a manufacturing process that produces a thin material diffusion zone at the junction which can be either $n /^{n+}$ or $p / p^{+}$. The thickness of the material diffusion zone impacts other device dimensions and should be small to increase thermal conductivity and overall performance. However, a very thin zone with a sharp transition from one material to another could adversely affect the $Z T$ coefficient because of the possible disruption of the crystal matrix. Thinness and high $Z T$ may require a tradeoff.
3. Estimate the thickness of the material diffusion zone.

4. Select the thicknesses of the thermoelectric layer $n$ and $n^{+}$or $p$ and $p^{+}$significantly (about 2 to 20 times) greater than the material diffusion zone. Thin thermoelectric layers increase thermal conductivity.

5. Select a dopant that maintains a high $Z T$. The dopant should minimally disturb the crystal matrix and have a relatively low ionization energy to embody the assumption made in the theory of a fermion gas minimally interacting with the matrix.

6. Select as high a doping level as possible to maximize heat flow by electrical carriers, but upwardly constrained:

(a) To keep the layer operating in the depletion zone throughout its whole thickness, to prevent space charges from cancelling the electrical field

(b) To keep the Fermi temperature near or below the input (e.g., ambient) temperature to enable the carrier's Fermi-Dirac statistics to differ markedly from the Maxwell-Boltzmann statistics 


\begin{tabular}{|c|c|}
\hline \multicolumn{2}{|c|}{ Electrical Insulator } \\
\hline$n x$ & \begin{tabular}{l|l}
$-\uparrow$ & $Q$
\end{tabular} \\
\hline ny & $+E \downarrow$ \\
\hline \multicolumn{2}{|c|}{ Electrical Insulator } \\
\hline px & $+{ }^{E} \mid Q$ \\
\hline py & $-\downarrow$ \\
\hline \multicolumn{2}{|c|}{ Electrical Insulator } \\
\hline$n x$ & -4 \\
\hline ny & $+E \downarrow$ \\
\hline \multicolumn{2}{|c|}{ Electrical Insulator } \\
\hline $\mathrm{px}$ & $+E \mid Q$ \\
\hline py & $-\downarrow \downarrow$ \\
\hline Electrical Ir & \\
\hline
\end{tabular}

(a)

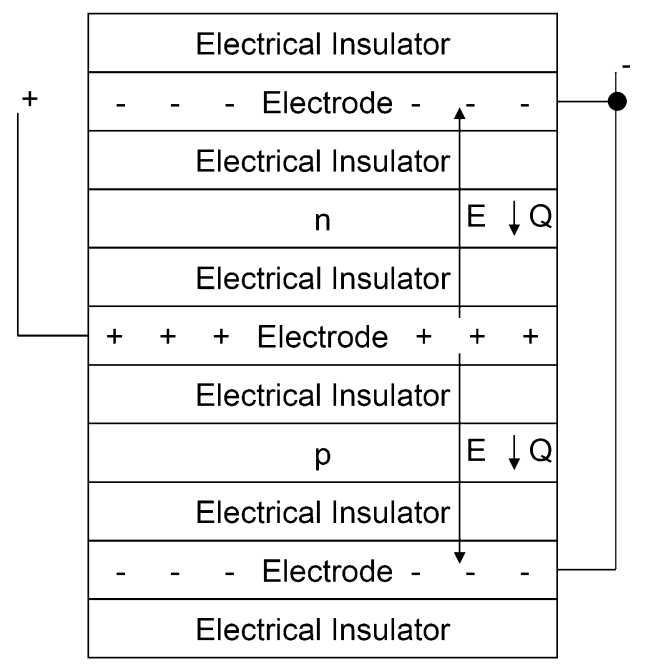

(b)

Fig. 8. (a) Interleaved and insulated heterogeneous $n$ junctions and $p$ junctions. The built-in field is generated by the different Fermi energies in the junction material. (b) Architecture in which the field is produced by insulated capacitive plates.

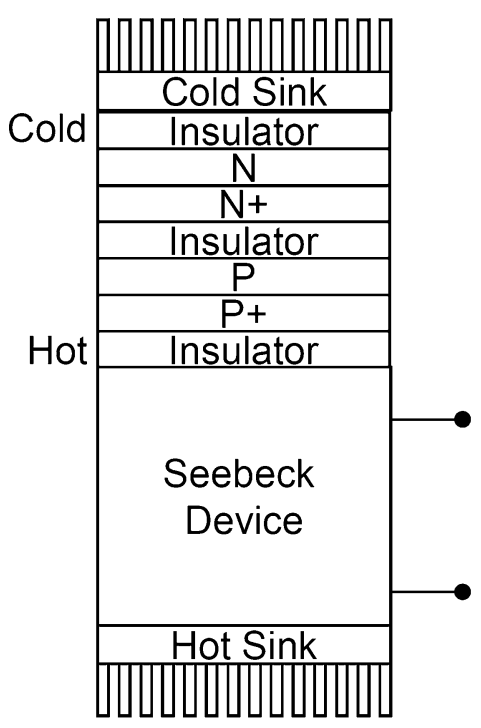

(a)

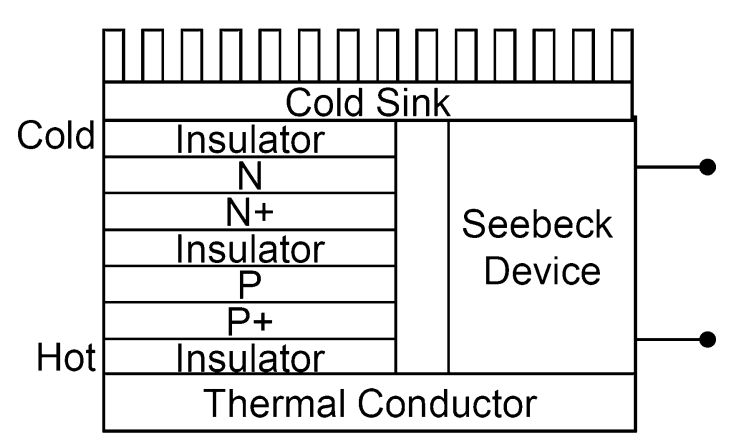

(b)

Fig. 9. (a) Series configuration. A Seebeck device is connected in series with a temperature generation device between a hot sink and a cold sink. (b) Folded design.

\section{PROPOSED APPLICATIONS AND ARCHITECTURES}

The proposed technology can be used in applications such as:

1. Refrigerators or heaters, in stand-alone mode, by promoting spontaneous heat flow from cold to hot without any energy input

2. Electrical generators when coupled with a Seebeck device, to convert ambient heat to electricity
3. Temperature boosters, to improving the performance of thermoelectric generators

4. Hybridized photovoltaics to provide electricity even at night.

The architectures presented in Figs. 9 and 10 combine temperature difference generators with Seebeck devices. 


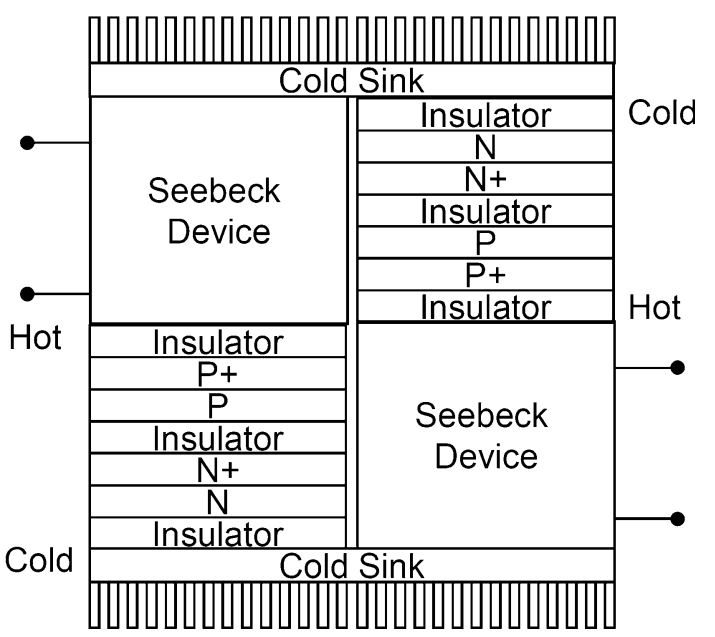

Fig. 10. Back-to-back configuration.

The temperature generation device in Fig. 9a comprises a stack of junctions, cold at the top and hot at the bottom.

The cold end of the stack is connected to a cold sink, resulting in the hot end of the stack being above ambient temperature. The Seebeck generator is connected to the hot end and, through a hot sink, to the environment. Heat flows from the cold end through the stack and into the Seebeck device, where it is partially converted to electrical power. The remainder of the heat is drained by the hot sink into the environment. Energy lost by the system is replaced by heat flowing into the cold end of the stack.

Figure 9b shows a folded design in which a Seebeck generator is connected across a stack of temperature generators.

A cold sink is attached to the cold end of the assembly to clamp the cold end to ambient temperature, resulting in the device operating above ambient. (Placing the cold sink at the hot end would have clamped the hot end to ambient, and resulted in the device operating below ambient.) The stack of junctions produces a temperature difference, hot at the bottom and cold at the top. Heat flows from the environment through the cold sink into the stack. Heat goes up the temperature gradient in the stack, then through the thermal conductor and into the Seebeck device, where some of the heat is converted into electrical energy. The remainder of the heat is drained at the cold sink at the top of the assembly.

Figure 10 shows two assemblies of Fig. 9b arranged back to back to boost the efficiencies of the Seebeck devices by draining their waste heat through cold sinks attached to the cold ends of the stacks.

\section{CONCLUSIONS}

Non-Maxwellian statistics are shown to produce a temperature gradient causing the Seebeck curve not to go through the origin and indicating an output voltage in the absence of an input temperature difference and an output temperature difference in the absence of an input current. This gradient is not observable in conventional semiconductors because it is shorted by heat phonons in the lattice. However, it has been detected in Seebeck measurements of high- $Z T$ materials.

Statistical temperature and kinetic temperature are identical in classical systems but differ in nonMaxwellian systems. Thermal equilibrium is found to be more generally defined by zero statistical temperature gradient, not necessarily by zero kinetic temperature gradient.

Temperature offsets in a thermoelectric device cannot be used to convert heat to work when the thermal carriers connecting the device to a heat engine are homogeneous with and indistinguishable from the carriers in the device. The temperature gradient produced by the thermal connection exactly cancels the gradient in the device. Similarly, the built-in potential of a junction cannot be used to produce work, because the potential produced by the leads exactly cancels the built-in potential. This is a consequence of the $H$-theorem, which assumes homogeneity and indistinguishability of particles. However, heterogeneous systems combine fermion statistics of electrical carriers and boson statistics of heat phonons and therefore fall outside of the coverage of the theorem. These systems can convert ambient heat to work.

Several thermoelectric architectures are suggested to test the theory. The architectures include homogeneous junctions, heterogeneous junctions, and capacitors. In addition, guidelines are also described, aimed at maximizing the measured temperature difference and power output.

A superlattice comprising layered temperature difference generators results in an amplified temperature difference. This architecture also boosts the Carnot efficiency and power output of a Seebeck device connected across the superlattice.

Iwanaga et al. and Snyder observed unexplained voltage and temperature offsets (Supplementary Material). They unsuccessfully tried to eliminate them using meticulous laboratory procedures, but did not attempt to maximize them. It is believed that, with better understanding of the theory, these offsets could be magnified by a factor of 2 to 10 . Assuming $0.2^{\circ} \mathrm{C}$ per junction, a 500-layer superlattice should conservatively produce a $100^{\circ} \mathrm{C}$ temperature difference that could be used by a Seebeck device to generate electricity. No input power is required. Therefore, if the device is used as a refrigerator, the coefficient of performance $(\mathrm{COP})=Q / W$ is infinite. Clearly in the context of this technology, the conventional concept of efficiency does not apply and should be replaced by an economic index such as fabrication dollar per watt output. The goal of the suggested guidelines is to boost this number. Further study is required to ascertain this cost. The construction of a successful working prototype will demonstrate the existence of 
a ubiquitous, abundant, and always available new source of energy.

\section{ACKNOWLEDGEMENTS}

I would like to thank my wife Penny for her unwavering support.

\section{CONFLICT OF INTEREST}

The author declares that he has no conflict of interest.

\section{ELECTRONIC SUPPLEMENTARY MATERIAL}

The online version of this article (doi: 10.1007/s11664-017-5875-0) contains supplementary material, which is available to authorized users.

\section{REFERENCES}

1. S. Iwanaga, E.S. Toberer, A. LaLonde, and G.J. Snyder, Rev. Sci. Instrum. 82, 063905-1-063905-6 (2011).

2. Y. Liu, C. Fu, H. Xie, and X. Zhao, J. Mater. Res. 30, 2670 (2015). doi:10.1557/jmr.2015.218.

3. J. Martin, Rev. Sci. Instrum. 83, 065101 (2012).

4. K.A. Borup, J. de Boor, H. Wang, F. Drymiotis, F. Gascoin, X. Shi, L. Chen, M.I. Fedorov, E. Muller, B.B. Iversen, and G.J. Snyder, Energy Environ. Sci. R. Soc. Chem. 8, 423 (2015).

5. G.S. Levy, Open Sci. J. Mod. Phys. 4(8), 1 (2017), https:// www.researchgate.net/publication/314899020. Accessed 19 Sept 2017.

6. G.S. Levy, Proceedings of the 11th International Conference on Ceramic Materials \& Components for Energy \& Environmental Applications, Vancouver, BC, Canada, 14-19 June 2015 Vol. 255 (Wiley: Hoboken, NJ, USA, 2016).

7. G.S. Levy, Entropy 17, 7645 (2015). doi:10.3390/e17117645.

8. G.S. Levy, J. Appl. Math. Phys. (2017). doi:10.4236/ jamp.2017.55102.

9. K.R. Narayanan and A.R. Srinivasa, Phys. Rev. E 85, 031151 (2017). doi:10.1103/PhysRevE.85.031151.
10. S. Ray, A. Baura, and B.C. Bag, Phys. Rev. E 89, 032148 (2014). doi:10.1103/PhysRevE.89.032148.

11. R.C. Tolman, The Principle of Statistical Mechanics, (Oxford at the Clarendon Press, 1938.) https://archive.org/ details/ThePrinciplesOfStatisticalMechanicsTolmanOxford AtTheClarendonPress1938. Accessed 5 June 2016.

12. G.S. Levy, Non-Maxwellian Distribution Calculator. http:// www.entropicpower.com. Accessed 25 September 2017.

13. G.S. Levy, Entropy 15, 4700 (2013). doi:10.3390/e15114700.

14. L. Boltzmann, Weitere Studien über das Wärmegleichgewicht unter Gasmolekülen, (Sitzungsberichte Akademie der Wissenschaften, 1872), 66; pp, 275-370. English translation: Boltzmann, L., Further Studies on the Thermal Equilibrium of Gas Molecules, The Kinetic Theory of Gases. History of Modern Physical Sciences 1. (2003) pp. 262-349.

15. J. Uffink, Compendium of the foundations of classical statistical physics, p. 44, 45 (2006) http://philsci-archive.pitt. edu/2691/1/UffinkFinal.pdf and http://philsci-archive.pitt. edu/2691/1/UffinkFinal.pdf. Accessed 16 September 2015).

16. H. Brown and W. Myrvold, (PhilSci-Archive. 09/2008. http:// www.socsci.uci.edu/ bskyrms/bio/readings/brown_myrvold_ on_boltzmann.pdf, also http://philsci-archive.pitt.edu/4187/1/ Reversibility08.pdf. Accessed 26 October 2013.

17. J. Von Neumann, Mathematical Foundations of Quantum Mechanics (Princeton: Princeton University Press, 1955).

18. J. Von Neumann, Proof of the Ergodic Theorem and the $H$ Theorem in Quantum Mechanics 10/5/1929; Translator: Roderich Tumulka, arXiv:1003.2133v2 (physics.hist-ph) 9 February 2010.

19. R. Clausius, Annalen der Physik 79: pp 368-397, 500-524 (1850). doi:10.1002/andp.18501550403. English translation in The London, Edinburgh and Dublin Philosophical Magazine and Journal of Science 4th 2(VIII): 1-21; 102-119.

20. V. Capeck and D. Sheehan, Challenges to the Second Law of Thermodynamics (Springer 2005), ISBN 1-4020-30150 (HB).

21. D.P. Sheehan, Phys. Rev. E 88, 032125 (2013).

22. D.P. Sheehan, Phys. Lett. A 280, 185 (2001).

23. D.P. Sheehan, D.J. Mallin, J.T. Garamella, and W.F. Sheehan, Found. Phys. 44, 235 (2014).

24. D.P. Sheehan, J.T. Garamella, D.J. Mallin, and W.F. Sheehan, R. Swed. Acad. Sci. Physica Scripta, (2012) T151.

25. D.P. Sheehan, T.A. Zawlacki, and W.H. Helmer, Rev. Sci. Instrum. 87, 074101 (2016).

26. R.W. Motley, Q-Machines (New York: Academic, 1975).

27. N. Rynn and N. D'Angelo, Rev. Sci. Instrum. 31, 6 (1960). doi:10.1063/1.1716884.

28. P.M. Orem and F.M. Orem, Entropy 19, 34 (2017). doi: 10.3390/e19010034. 